\title{
Fibonacci collocation method for solving a class of systems of nonlinear differential equations
}

\author{
Sertan Alkan ${ }^{1, *}$ and Musa Cakmak ${ }^{2}$ \\ ${ }^{1}$ Department of Computer Engineering, Iskenderun Technical University, Hatay, Turkey \\ ${ }^{2}$ Department of Accounting and Tax Applications, Hatay Mustafa Kemal University, Hatay, Turkey
}

Received: 20 October 2021, Accepted: 19 December 2021

Published online: 23 December 2021.

\begin{abstract}
In this study, we present an approximate solution of a class of systems of nonlinear differential equations using Fibonacci collocation method. The problem is firstly reduced into a nonlinear algebraic system, later the unknown coefficients of the approximate solution function are obtained. Also, some test problems are given to illustrate the effective of the proposed method. Finally, the obtained numerical results are compared with exact solutions of the test problems and approximate ones obtained with the Chebyshev operational matrix method.
\end{abstract}

Keywords: The systems of nonlinear differential equations, Collocation method, Fibonacci polynomials.

\section{Introduction}

Finding the solution of nonlinear differential equations is quite important because these equations are often used to model scientific phenomena. For this reason, many methods have been developed for solving these equations approximately. Some of these methods are as follows: Hermite wavelet method [1], homotopy analysis method [2], Laguerre collocation method [3], variational iteration method [4], optimized decomposition method [5].

In [6], the approximate solutions of a class of systems of linear Fredholm integro-differential equations is obtained using Fibonacci collocation method. Similarly, in [7], the high-order linear Fredholm integro-differential-difference equations are approximately solved by using Fibonacci collocation method. Also, in [8], Fibonacci collocation method is used for approximately solving a class of systems of high-order linear Volterra integro-differential equations. In [9], Fibonacci collocation method is applied to linear differential-difference equations. The paper given by [10] deals with that the application of Fibonacci collocation method to singularly perturbed differential-difference equations.

In this paper, the Fibonacci collocation method is firstly used for solving the following class of systems of nonlinear differential equation:

$$
\begin{gathered}
\sum_{k=0}^{2} \sum_{r=1}^{2} P_{j k r}(x) u_{r}^{(k)}(x)+\sum_{k=0}^{2} \sum_{r=1}^{2} Q_{j k r s p}(x) u_{s}^{r}(x) u_{p}^{(k)}(x)=g_{j}(x) \\
0 \leq x \leq 1, j, s, p=1,2
\end{gathered}
$$

with the initial conditions

$$
\sum_{k=0}^{1}\left[a_{j k} u_{r}^{(k)}(0)+b_{j k} u_{r}^{(k)}(0)\right]=\delta_{j r}, \quad j=1,2
$$


where $u_{r}^{(0)}(x)=u_{r}(x), u_{r}^{0}(x)=1$ and $u_{r}(x)$ is an unknown functions. $P_{j k r}(x), Q_{j k r s p}(x)$ and $g_{j}(x)$ are given continuous functions on interval $[0,1], a_{j k}, b_{j k}$, and $\delta_{j r}$ are suitable constants. The aim of this study is to get the approximate solutions as the truncated Fibonacci series defined by

$$
u_{r}(x)=\sum_{n=1}^{N+1} c_{r n} F_{n}(x)
$$

where $F_{n}(x)$ denotes the Fibonacci polynomials; $c_{r n}(1 \leq r n \leq N+1)$ are unknown Fibonacci polynomial coefficients, and $N$ is any positive integer such that $N \geq m$.

The paper consists of 6 sections. In Section 2, the properties and definitions related to Fibonacci polynomials are given. In Section 3, the fundamental matrix forms of Fibonacci collocation method by using fundamental relations of Fibonacci polynomials are constructed. In section 4 , the absolute error function is formulated. In Section 5 , test problems are presented and the proposed method are tested. Finally, conclusions are presented in Section 6.

\section{Properties of Fibonacci polynomials}

The Fibonacci polynomials were studied by Falcon and Plaza [11,12]. The recurrence relation of those polynomials is defined by

$$
F_{n}(x)=x F_{n-1}(x)+F_{n-2}(x)
$$

For $n \geqslant 3$. , $F_{1}(x)=1, F_{2}(x)=x$. The properties were further investigated by Falcon and Plaza in [11,12]. The first few Fibonacci polynomials are

$$
\begin{aligned}
& F_{1}=1, \\
& F_{2}=x, \\
& F_{3}=x^{2}+1, \\
& F_{4}=x^{3}+2 x, \\
& F_{5}=x^{4}+3 x^{2}+1, \\
& F_{6}=x^{5}+4 x^{3}+3 x, \\
& F_{7}=x^{6}+5 x^{4}+6 x^{2}+1, \\
& F_{8}=x^{7}+6 x^{5}+10 x^{3}+4 x,
\end{aligned}
$$

\section{Fundamental relations}

Let us assume that linear combination of Fibonacci polynomials (3) is an approximate solutions of Eq (1). Our purpose is to determine the matrix forms of Eq (1) by using (3). Firstly, we can write Fibonacci polynomials (5) in the matrix form

$$
\mathbf{F}(x)=\mathbf{T}(x) \mathbf{M}
$$




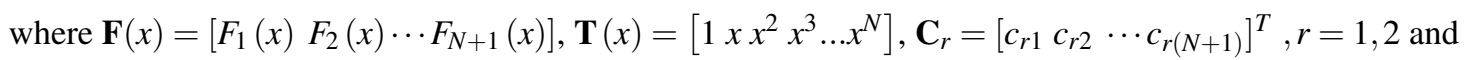

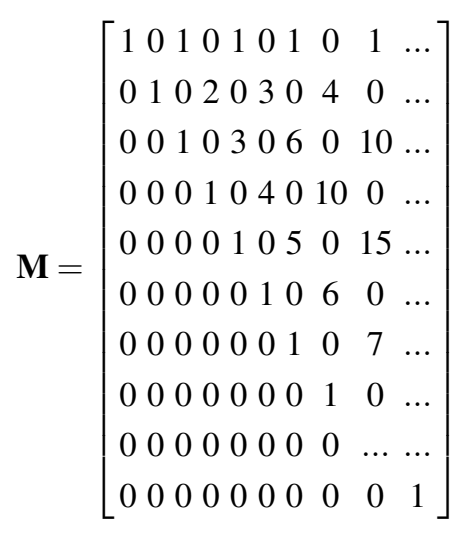

Then we set the approximate solutions defined by a truncated Fibonacci series (3) in the matrix form

$$
u_{r}(x)=\mathbf{F}(x) \mathbf{C}_{r} .
$$

By using (6) and (7), the matrix relation is expressed as

$$
\begin{aligned}
u_{r}(x) & \cong u_{r N}(x)=\mathbf{F}(x) \mathbf{C}_{r}=\mathbf{T}(x) \mathbf{M \mathbf { C } _ { r }} \\
u_{r}^{\prime}(x) & \cong u_{r N}^{\prime}(x)=\mathbf{T B M C} \mathbf{C}_{r} \\
u_{r}^{\prime \prime}(x) & \cong u_{r N}^{\prime \prime}(x)=\mathbf{T}(x) \mathbf{B}^{2} \mathbf{M} \mathbf{C}_{r} \\
\ldots & \\
u_{r}^{(k)}(x) & \cong u_{r N}^{(k)}(x)=\mathbf{T}(x) \mathbf{B}^{k} \mathbf{M} \mathbf{C}_{r}
\end{aligned}
$$

where $r=1,2$. Also, the relations between the matrix $\mathbf{T}(x)$ and its derivatives, $\mathbf{T}^{\prime}(x), \mathbf{T}^{\prime \prime}(x), \ldots, \mathbf{T}^{(k)}(x)$ are

$$
\begin{aligned}
\mathbf{T}^{\prime}(x) & =\mathbf{T}(x) \mathbf{B}, \mathbf{T}^{\prime \prime}(x)=\mathbf{T}(x) \mathbf{B}^{2} \\
\mathbf{T}^{\prime \prime \prime}(x) & =\mathbf{T}(x) \mathbf{B}^{3}, \ldots, \mathbf{T}^{(k)}(x)=\mathbf{T}(x) \mathbf{B}^{k}
\end{aligned}
$$

Then we set the approximate solution defined by a truncated Fibonacci series (3) in the matrix form

$$
u_{r}(x) \cong u_{r N}(x)=\mathbf{F}(x) \mathbf{C}_{r} .
$$

By substituting the Fibonacci collocation points defined by

$$
x_{i}=\frac{i}{N}, i=0,1, \ldots N
$$

into $\mathrm{Eq}(8)$, we have

$$
u_{r}^{(k)}\left(x_{i}\right)=\mathbf{T}\left(x_{i}\right) \mathbf{B}^{k} \mathbf{M} \mathbf{C}_{r} .
$$

and the compact form of the relation (12) becomes

$$
\mathbf{U}_{r}^{(k)}=\mathbf{T B}^{k} \mathbf{M C}_{r}, \quad k=0,1,2, \quad r=1,2
$$


where

$$
\begin{aligned}
& \mathbf{U}_{r}^{(k)}=\left[\begin{array}{c}
u_{r}^{(k)}\left(x_{0}\right) \\
u_{r}^{(k)}\left(x_{1}\right) \\
\vdots \\
u_{r}^{(k)}\left(x_{N}\right)
\end{array}\right] \\
& \mathbf{B}=\left[\begin{array}{cccccccc}
0 & 1 & 0 & 0 & 0 & 0 & \cdots & 0 \\
0 & 0 & 2 & 0 & 0 & 0 & \cdots & 0 \\
0 & 0 & 0 & 3 & 0 & 0 & \cdots & 0 \\
0 & 0 & 0 & 0 & 4 & 0 & \cdots & 0 \\
0 & 0 & 0 & 0 & 0 & 5 & \cdots & 0 \\
0 & 0 & 0 & 0 & 0 & 0 & \cdots & 0 \\
\vdots & \vdots & \vdots & \vdots & \vdots & \ddots & \ddots & N \\
0 & 0 & 0 & 0 & 0 & 0 & \cdots & 0
\end{array}\right], \mathbf{B}^{0}=\left[\begin{array}{cccccccc}
1 & 0 & 0 & 0 & 0 & 0 & \cdots & 0 \\
0 & 1 & 0 & 0 & 0 & 0 & \cdots & 0 \\
0 & 0 & 1 & 0 & 0 & 0 & \cdots & 0 \\
0 & 0 & 0 & 1 & 0 & 0 & \cdots & 0 \\
0 & 0 & 0 & 0 & 1 & 0 & \cdots & 0 \\
0 & 0 & 0 & 0 & 0 & 1 & \cdots & 0 \\
\vdots & \vdots & \vdots & \vdots & \vdots & \ddots & \ddots & 0 \\
0 & 0 & 0 & 0 & 0 & 0 & \cdots & 1
\end{array}\right] \\
& \mathbf{T}=\left[\begin{array}{c}
\mathbf{T}\left(x_{0}\right) \\
\mathbf{T}\left(x_{1}\right) \\
\vdots \\
\mathbf{T}\left(x_{N}\right)
\end{array}\right]=\left[\begin{array}{cccc}
1 & x_{0} & \ldots & x_{0}^{N} \\
1 & x_{1} & \ldots & x_{1}^{N} \\
1 & \vdots & \ldots & \vdots \\
1 & x_{N} & \ldots & x_{N}^{N}
\end{array}\right]
\end{aligned}
$$

In addition, we can obtain the matrix form $\left(\hat{\mathbf{U}}_{s}\right)^{r} \hat{\mathbf{U}}_{p}^{(k)}$ which appears in the nonlinear part of Eq. (1), by using Eq. (8) as

$$
\left(\hat{\mathbf{U}}_{s}\right)^{r} \hat{\mathbf{U}}_{p}^{(k)}=\left[\begin{array}{c}
u_{s}^{r}\left(x_{0}\right) u_{p}^{(k)}\left(x_{0}\right) \\
u_{s}^{r}\left(x_{1}\right) u_{p}^{(k)}\left(x_{1}\right) \\
\vdots \\
u_{s}^{r}\left(x_{N}\right) u_{p}^{(k)}\left(x_{N}\right)
\end{array}\right]=\left[\begin{array}{cccc}
u_{s}\left(x_{0}\right) & 0 & \ldots & 0 \\
0 & u_{s}\left(x_{1}\right) & \ldots & 0 \\
\vdots & \vdots & \ddots & \vdots \\
0 & 0 & \ldots & u_{s}\left(x_{N}\right)
\end{array}\right]^{r}\left[\begin{array}{c}
u_{p}^{(k)}\left(x_{0}\right) \\
u_{p}^{(k)}\left(x_{1}\right) \\
\vdots \\
u_{p}^{(k)}\left(x_{N}\right)
\end{array}\right]
$$

where

$$
\begin{gathered}
\left(\hat{\mathbf{U}}_{s}\right)^{r} \hat{\mathbf{U}}_{p}^{(k)}=\left(\hat{\mathbf{T}} \hat{\mathbf{M}} \hat{\mathbf{C}}_{r}\right)^{r} \mathbf{T}(\mathbf{B})^{\mathbf{k}} \mathbf{M} . \\
\hat{\mathbf{T}}=\left[\begin{array}{cccc}
\mathbf{T}\left(x_{0}\right) & 0 & \ldots & 0 \\
0 & \mathbf{T}\left(x_{1}\right) & \ldots & 0 \\
\vdots & \vdots & \ddots & \vdots \\
0 & 0 & \ldots & \mathbf{T}\left(x_{N}\right)
\end{array}\right], \hat{\mathbf{B}}=\left[\begin{array}{cccc}
\mathbf{B} & 0 & \ldots & 0 \\
0 & \mathbf{B} & \ldots & 0 \\
\vdots & \vdots & \ddots & \vdots \\
0 & 0 & \ldots & \mathbf{B}
\end{array}\right], \\
\hat{\mathbf{M}}=\left[\begin{array}{cccc}
\mathbf{M} & 0 & \ldots & 0 \\
0 & \mathbf{M} & \ldots & 0 \\
\vdots & \vdots & \ddots & \vdots \\
0 & 0 & \ldots & \mathbf{M}
\end{array}\right], \hat{\mathbf{C}}_{r}=\left[\begin{array}{cccc}
\mathbf{C}_{r} & 0 & \ldots & 0 \\
0 & \mathbf{C}_{r} & \ldots & 0 \\
\vdots & \vdots & \ddots & \vdots \\
0 & 0 & \ldots & \mathbf{C}_{r}
\end{array}\right]
\end{gathered}
$$


Substituting the collocation points $\left(x_{i}=i / N, i=0,1, \cdots, N\right)$ into Eq. (1), gives the system of equations

$$
\sum_{k=0}^{2} \sum_{r=1}^{2} P_{j k r}\left(x_{i}\right) u_{r}^{(k)}\left(x_{i}\right)+\sum_{k=0}^{2} \sum_{r=1}^{2} Q_{j k r s p}\left(x_{i}\right) u_{s}^{r}\left(x_{i}\right) u_{p}^{(k)}\left(x_{i}\right)=g_{j}\left(x_{i}\right), \quad 0 \leq x \leq 1
$$

which can be expressed with the aid of Eqs. (14) and (??) as

$$
\sum_{k=0}^{2} \sum_{r=1}^{2} P_{j k r} \mathbf{U}_{r}^{(k)}+\sum_{k=0}^{2} \sum_{r=1}^{2} Q_{j k r s p}\left(\hat{\mathbf{U}}_{s}\right)^{r} \hat{\mathbf{U}}_{p}^{(k)}=\mathbf{G}_{j}
$$

where

$$
\begin{aligned}
P_{j k r} & =\operatorname{diag}\left[\begin{array}{llll}
P_{j k r}\left(x_{0}\right) & P_{j k r}\left(x_{1}\right) & \ldots & P_{j k r}\left(x_{N}\right)
\end{array}\right], \\
Q_{j k r s p} & =\operatorname{diag}\left[\begin{array}{llll}
Q_{j k r s p}\left(x_{0}\right) & Q_{j k r s p}\left(x_{1}\right) & \ldots & Q_{j k r s p}\left(x_{N}\right)
\end{array}\right] \\
\mathbf{G}_{j} & =\left[\begin{array}{llll}
g j\left(x_{0}\right) & g_{j}\left(x_{1}\right) & \ldots & g_{j}\left(x_{N}\right)
\end{array}\right]^{T}, j=1,2 .
\end{aligned}
$$

Substituting the relations (13) and (15) into Eq. (16), the fundamental matrix equation can be obtained as

$$
\left\{\sum_{k=0}^{2} \sum_{r=1}^{2} P_{j k r} \mathbf{T B}^{k} \mathbf{M}+\sum_{k=0}^{2} \sum_{r=1}^{2} Q_{j k r s p}\left(\hat{\mathbf{T}} \hat{\mathbf{M}} \hat{\mathbf{C}}_{r}\right)^{r} \mathbf{T}(\mathbf{B})^{\mathbf{k}} \mathbf{M}\right\} \mathbf{C}_{r}=\mathbf{G}_{j}
$$

Shortly, Eq. (17) is also written in the following form

$$
\mathbf{W C}=\mathbf{G} \quad \text { or }[\mathbf{W} ; \mathbf{G}]
$$

where

$$
\begin{aligned}
\mathbf{W} & =\left[\begin{array}{ll}
W_{11} & W_{12} \\
W_{21} & W_{22}
\end{array}\right], \mathbf{C}=\left[\begin{array}{l}
\mathbf{C}_{1} \\
\mathbf{C}_{2}
\end{array}\right], \mathbf{G}=\left[\begin{array}{l}
\mathbf{G}_{1} \\
\mathbf{G}_{2}
\end{array}\right] \\
W_{11} & =\sum_{k=0}^{2} \sum_{r=1}^{1} P_{j k r} \mathbf{T} \mathbf{B}^{k} \mathbf{M}+\sum_{k=0}^{2} \sum_{r=1}^{1} Q_{j k r s p}\left(\hat{\mathbf{T}} \hat{\mathbf{M}} \hat{\mathbf{C}}_{r}\right)^{r} \mathbf{T}(\mathbf{B})^{\mathbf{k}} \mathbf{M} \quad \text { for } \mathbf{j}=\mathbf{1} \\
W_{12} & =\sum_{k=0}^{2} \sum_{r=2}^{2} P_{j k r} \mathbf{T} \mathbf{B}^{k} \mathbf{M}+\sum_{k=0}^{2} \sum_{r=2}^{2} Q_{j k r s p}\left(\hat{\mathbf{T}} \hat{\mathbf{M}} \hat{\mathbf{C}}_{r}\right)^{r} \mathbf{T}(\mathbf{B})^{\mathbf{k}} \mathbf{M} \text { for } \mathbf{j}=\mathbf{1} \\
W_{21} & =\sum_{k=0}^{2} \sum_{r=1}^{1} P_{j k r} \mathbf{T} \mathbf{B}^{k} \mathbf{M}+\sum_{k=0}^{2} \sum_{r=1}^{1} Q_{j k r s p}\left(\hat{\mathbf{T}} \hat{\mathbf{M}} \hat{\mathbf{C}}_{r}\right)^{r} \mathbf{T}(\mathbf{B})^{\mathbf{k}} \mathbf{M} \text { for } \mathbf{j}=\mathbf{2} \\
W_{12} & =\sum_{k=0}^{2} \sum_{r=2}^{2} P_{j k r} \mathbf{T} \mathbf{B}^{k} \mathbf{M}+\sum_{k=0}^{2} \sum_{r=2}^{2} Q_{j k r s p}\left(\hat{\mathbf{T}} \hat{\mathbf{M}} \hat{\mathbf{C}}_{r}\right)^{r} \mathbf{T}(\mathbf{B})^{\mathbf{k}} \mathbf{M} \text { for } \mathbf{j}=\mathbf{2} .
\end{aligned}
$$

Here, Eq. (18) is a system containing $(N+1)$ linear algebraic equations with the $(N+1)$ unknown Fibonacci coefficients $c_{r n}, n=1,2, \ldots, N+1$. Using Eq. (13) at the point 0, the matrix representation of the initial conditions in Eq. (2) is given by

$$
\left\{\sum_{k=0}^{m-1}\left[a_{j k} \mathbf{T}(0)+b_{j k} \mathbf{T}(0)\right](\mathbf{B})^{(k)} \mathbf{M}\right\} \mathbf{C}_{r}=\delta_{j r}, j=0,1,2, \ldots, m-1
$$

or briefly

$$
\mathbf{V}_{j r} \mathbf{C}_{r}=\left[\boldsymbol{\delta}_{j r}\right] \quad \text { or } \quad\left[\mathbf{V}_{j r} ; \delta_{j r}\right] ; \quad j=0,1,2, \ldots, m-1
$$


where

$$
\mathbf{V}_{j r}=\sum_{k=0}^{m-1}\left[a_{j k} \mathbf{T}(0)+b_{j k} \mathbf{T}(0)\right](\mathbf{B})^{(k)} \mathbf{M}=\left[\begin{array}{lllll}
v_{j o} & v_{j 1} & v_{j 2} & \ldots & v_{j N}
\end{array}\right] .
$$

Therefore, by replacing the row matrices in (19) by the last $m$ rows of the augmented matrix (18), the new augmented matrix becomes

$$
\hat{\mathbf{W}} \mathbf{C}=\hat{\mathbf{G}} \text { or }[\hat{\mathbf{W}} ; \hat{\mathbf{G}}]
$$

which is an algebraic system. Here,

$$
[\hat{\mathbf{W}} ; \hat{\mathbf{G}}]=\left[\begin{array}{ll}
\hat{W}_{11} & \hat{W}_{12} \\
\hat{W}_{21} & \hat{W}_{22}
\end{array}\right]
$$

where

$$
\left[\hat{W}_{11}\right]=\left[\begin{array}{ccccc}
w_{11} & w_{12} & w_{13} & \cdots & w_{1 N+1} \\
w_{21} & w_{22} & w_{23} & \cdots & w_{2 N+1} \\
w_{31} & w_{32} & w_{33} & \cdots & w_{3 N+1} \\
\vdots & \vdots & \vdots & \ddots & \vdots \\
w_{(N+1-m) 1} & w_{(N+1-m) 2} & w_{(N+1-m) 3} & \cdots & w_{(N+1-m) N+1} \\
v_{11} & v_{12} & v_{13} & \cdots & v_{1 N+1} \\
v_{21} & v_{22} & v_{23} & \cdots & v_{2 N+1} \\
v_{31} & v_{32} & v_{33} & \cdots & v_{3 N+1} \\
\vdots & \vdots & \vdots & \ddots & \vdots \\
v_{(m-1) 1} & v_{(m-1) 2} & v_{(m-1) 3} & \cdots & v_{(m-1) N+1}
\end{array}\right]
$$




$$
\begin{aligned}
& {\left[\hat{W}_{21}\right]=\left[\begin{array}{ccccc}
w_{11} & w_{12} & w_{13} & \cdots & w_{1 N+1} \\
w_{21} & w_{22} & w_{23} & \cdots & w_{2 N+1} \\
w_{31} & w_{32} & w_{33} & \cdots & w_{3 N+1} \\
\vdots & \vdots & \vdots & \ddots & \vdots \\
w_{(N+1-m) 1} & w_{(N+1-m) 2} & w_{(N+1-m) 3} & \cdots & w_{(N+1-m) N+1} \\
0 & 0 & 0 & \cdots & 0 \\
0 & 0 & 0 & \cdots & 0 \\
0 & 0 & 0 & \cdots & 0 \\
\vdots & \vdots & \vdots & \ddots & \vdots \\
0 & 0 & 0 & \cdots & 0
\end{array}\right]} \\
& {\left[\hat{W}_{22}\right]=\left[\begin{array}{ccccc}
w_{11} & w_{12} & w_{13} & \cdots & w_{1 N+1} \\
w_{21} & w_{22} & w_{23} & \cdots & w_{2 N+1} \\
w_{31} & w_{32} & w_{33} & \cdots & w_{3 N+1} \\
\vdots & \vdots & \vdots & \ddots & \vdots \\
w_{(N+1-m) 1} & w_{(N+1-m) 2} & w_{(N+1-m) 3} & \cdots & w_{(N+1-m) N+1} \\
v_{11} & v_{12} & v_{13} & \cdots & v_{1 N+1} \\
v_{21} & v_{22} & v_{23} & \cdots & v_{2 N+1} \\
v_{31} & v_{32} & v_{33} & \cdots & v_{3 N+1} \\
\vdots & \vdots & \vdots & \ddots & \vdots \\
v_{(m-1) 1} & v_{(m-1) 2} & v_{(m-1) 3} & \cdots & v_{(m-1) N+1}
\end{array}\right]} \\
& \hat{\mathbf{G}}=\left[\begin{array}{l}
\hat{\mathbf{G}}_{1} \\
\hat{\mathbf{G}}_{2}
\end{array}\right]
\end{aligned}
$$

where

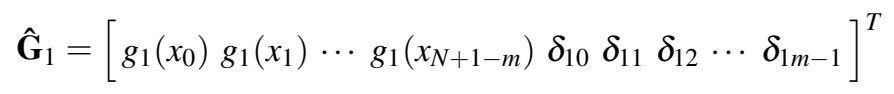

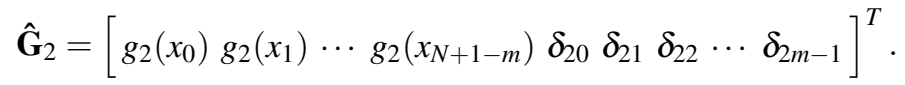

In this way, by solving the linear equation system in (20), the unknown Fibonacci coefficients $c_{r n}, n=1,2, \ldots, N+1$ are calculated and substituted into (3), and the approximate solution is obtained.

\section{Error Estimation}

In this section, it is given that the error function $E_{i, N}(x)$ for $i=1,2$ to test the accuracy of the proposed method. The function $E_{i, N}(x)$ is given by

$$
E_{i, N}(x)=\left|u_{i, N}(x)-u_{i}(x)\right| \text { for } i=1,2
$$

where $u_{i, N}(x)$ and $u_{i}(x)$ are the approximate and exact solutions of Eq.(1), respectively. 


\section{Numerical examples}

In this section, three numerical examples are given to show the efficient of the proposed method. On these problems, the method is tested by using the error function given by (21).

Example 1. Consider the nonlinear differential equation

$$
\begin{gathered}
u_{1}^{\prime}(x)+u_{2}^{\prime}(x)+u_{2}(x)+u_{1}^{\prime}(x) u_{2}(x)=2 x^{2}-3 x-2 \\
u_{1}^{\prime}(x)-x u_{2}^{\prime}(x)+u_{1}(x)+u_{1}^{2}(x)=x^{4}+x^{2}+x
\end{gathered}
$$

with initial conditions

$$
u_{1}(0)=0, u_{2}(0)=-3
$$

and the exact solutions $u_{1}(x)=x^{2}, u_{2}(x)=x-3$. The approximate the solution $u_{r}(x)$ by the Fibonacci polynomials is

$$
u_{r}(x)=\sum_{n=1}^{N+1} c_{r n} F_{n}(x)
$$

where $N=2, P_{111}(x)=1, P_{112}(x)=1, P_{102}(x)=1, Q_{10112}(x)=1, g_{1}(x)=2 x^{2}-3 x-2$, and $P_{211}(x)=1, P_{212}(x)=$ $-x, P_{201}(x)=1,, Q_{2011}(x)=1, g_{2}(x)=x^{4}+x^{2}+x$. Hence, the set of collocation points (11) for $N=2$ is computed as

$$
x_{0}=0, x_{1}=\frac{1}{2}, x_{2}=1
$$

From Eq. (17), the fundamental matrix equation of the problem is

$$
\begin{aligned}
& \mathbf{G}_{1}=\left\{\mathbf{P}_{111} \mathbf{T B M}+\mathbf{Q}_{10111} \hat{\mathbf{T}} \hat{\mathbf{M}} \hat{\mathbf{C}}_{2} \mathbf{T} \mathbf{B M}\right\} \mathbf{C}_{1}+\left\{\mathbf{P}_{112} \mathbf{T B M}+\mathbf{P}_{102} \mathbf{T M}\right\} \mathbf{C}_{2} \\
& \mathbf{G}_{2}=\left\{\mathbf{P}_{211} \mathbf{T B M}+\mathbf{P}_{201} \mathbf{T M}+\mathbf{Q}_{2011} \hat{\mathbf{T}} \hat{\mathbf{M}} \hat{\mathbf{C}}_{1} \mathbf{T} \mathbf{M}\right\} \mathbf{C}_{1}+\left\{\mathbf{P}_{212} \mathbf{T B M}\right\} \mathbf{C}_{2}
\end{aligned}
$$

where

$$
\begin{gathered}
\mathbf{W}_{11}=\mathbf{P}_{111} \mathbf{T B M}+\mathbf{Q}_{10111} \hat{\mathbf{T}} \hat{\mathbf{M}} \hat{\mathbf{C}}_{2} \mathbf{T} \mathbf{B M} \\
\mathbf{W}_{12}=\mathbf{P}_{112} \mathbf{T B M}+\mathbf{P}_{102} \mathbf{T M} \\
\mathbf{W}_{21}=\mathbf{P}_{211} \mathbf{T B M}+\mathbf{P}_{201} \mathbf{T M}+\mathbf{Q}_{20111} \hat{\mathbf{T}} \hat{\mathbf{M}} \hat{\mathbf{C}}_{1} \mathbf{T} \mathbf{M} \\
\mathbf{W}_{22}=\mathbf{P}_{212} \mathbf{T B M} \\
\mathbf{P}_{111}=\mathbf{P}_{112}=\mathbf{P}_{102}=\mathbf{Q}_{10112}=\left[\begin{array}{lll}
1 & 0 & 0 \\
0 & 1 & 0 \\
0 & 0 & 1
\end{array}\right], \mathbf{P}_{211}=\mathbf{P}_{201}=\mathbf{Q}_{20111}=\left[\begin{array}{lll}
1 & 0 & 0 \\
0 & 1 & 0 \\
0 & 0 & 1
\end{array}\right], \mathbf{P}_{212}=\left[\begin{array}{ccc}
0 & 0 & 0 \\
0 & \frac{-1}{2} & 0 \\
0 & 0 & -1
\end{array}\right], \\
\mathbf{T}=\left[\begin{array}{c}
\mathbf{T}(0) \\
\mathbf{T}\left(\frac{1}{2}\right) \\
\mathbf{T}(1)
\end{array}\right]=\left[\begin{array}{lll}
1 & 0 & 0 \\
1 & \frac{1}{2} & \frac{1}{4} \\
1 & 1 & 1
\end{array}\right], \mathbf{M}=\left[\begin{array}{lll}
1 & 0 & 1 \\
0 & 1 & 0 \\
0 & 0 & 1
\end{array}\right], \mathbf{B}=\left[\begin{array}{lll}
0 & 1 & 0 \\
0 & 0 & 2 \\
0 & 0 & 0
\end{array}\right],
\end{gathered}
$$




$$
\begin{aligned}
& \hat{\mathbf{T}}=\left[\begin{array}{cccc}
\mathbf{T}\left(x_{0}\right) & 0 & \ldots & 0 \\
0 & \mathbf{T}\left(x_{1}\right) & \ldots & 0 \\
\vdots & \vdots & \ddots & \vdots \\
0 & 0 & \ldots & \mathbf{T}\left(x_{N}\right)
\end{array}\right]=\left[\begin{array}{lllllllll}
1 & 0 & 0 & 0 & 0 & 0 & 0 & 0 & 0 \\
0 & 0 & 0 & 1 & 1 & \frac{1}{2} & 0 & 0 & 0 \\
0 & 0 & 0 & 0 & 0 & 0 & 1 & 1 & 1
\end{array}\right] \\
& \hat{\mathbf{M}}=\left[\begin{array}{cccc}
\mathbf{M} & 0 & \ldots & 0 \\
0 & \mathbf{M} & \ldots & 0 \\
\vdots & \vdots & \ddots & \vdots \\
0 & 0 & \ldots & \mathbf{M}
\end{array}\right], \hat{\mathbf{C}}_{r}=\left[\begin{array}{cccc}
\mathbf{C}_{r} & 0 & \ldots & 0 \\
0 & \mathbf{C}_{r} & \ldots & 0 \\
\vdots & \vdots & \ddots & \vdots \\
0 & 0 & \ldots & \mathbf{C}_{r}
\end{array}\right] \\
& \mathbf{W}=\left[\begin{array}{ll}
W_{11} & W_{12} \\
W_{21} & W_{22}
\end{array}\right], \mathbf{C}_{1}=\left[\begin{array}{lll}
a & b & c
\end{array}\right]^{T}, \mathbf{C}_{2}=\left[\begin{array}{lll}
k l m
\end{array}\right]^{T}, \mathbf{C}=\left[\begin{array}{l}
\mathbf{C}_{1} \\
\mathbf{C}_{2}
\end{array}\right] \\
& \mathbf{G}=\left[\begin{array}{l}
\mathbf{G}_{1} \\
\mathbf{G}_{2}
\end{array}\right], \mathbf{G}_{1}=\left[\begin{array}{lll}
-2 & -3-3
\end{array}\right]^{T}, \mathbf{G}_{2}=\left[\begin{array}{lll}
0 & \frac{13}{19} & 3
\end{array}\right]^{T}, \\
& W_{11}=\left[\begin{array}{ccc}
0 & k+m+1 & 0 \\
0 & k+\frac{1}{2} l+\frac{5}{4} m+1 & k+\frac{1}{2} l+\frac{5}{4} m+1 \\
0 & k+l+2 m+1 & 2 k+2 l+4 m+2
\end{array}\right], W_{12}=\left[\begin{array}{lll}
1 & 1 & 1 \\
1 & \frac{3}{2} & \frac{9}{4} \\
1 & 2 & 4
\end{array}\right], \\
& W_{21}=\left[\begin{array}{ccc}
a+c+1 & 1 & a+c+1 \\
a+\frac{1}{2} b+\frac{5}{4} c+1 & \frac{1}{2} a+\frac{1}{4} b+\frac{5}{8} c+\frac{3}{2} & \frac{5}{4} a+\frac{5}{8} b+\frac{25}{16} c+\frac{9}{4} \\
a+b+2 c+1 & a+b+2 c+2 & 2 a+2 b+4 c+4
\end{array}\right], W_{22}=\left[\begin{array}{ccc}
0 & 0 & 0 \\
0 & -\frac{1}{2} & -\frac{1}{2} \\
0 & -1 & -2
\end{array}\right],
\end{aligned}
$$

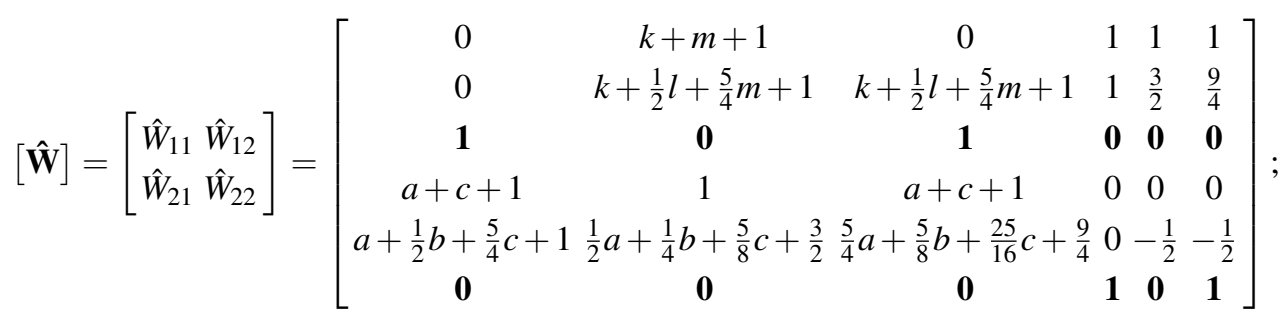

$$
\begin{aligned}
& \hat{\mathbf{G}}=\left[\begin{array}{llllll}
-2 & -3 & \mathbf{0} & 0 & \frac{13}{16} & -\mathbf{3}
\end{array}\right]^{T}
\end{aligned}
$$

From Eq. (19), the matrix form for initial condition is

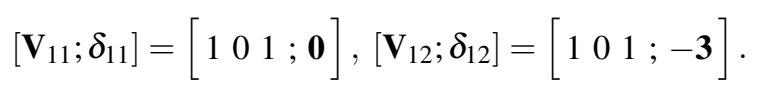


Consequently, by solving the system $[\hat{\mathbf{W}} ; \hat{\mathbf{G}}]$, the Fibonacci coefficients matrix are obtained

$$
\mathbf{C}=\left[\begin{array}{lllll}
-1 & 0 & 1-3 & 1 & 0
\end{array}\right]^{T}
$$

where

$$
\mathbf{C}_{1}=\left[\begin{array}{lll}
-1 & 0 & 1
\end{array}\right]^{T}, \mathbf{C}_{2}=\left[\begin{array}{lll}
-3 & 1 & 0
\end{array}\right]^{T}
$$

The approximate solutions for $N=2$ in terms of the Fibonacci polynomials is obtained as

$$
u_{1}(x)=x^{2} \text { and } u_{2}(x)=x-3
$$

Example 2. [13] Consider that the following differential equation system

$$
\left\{\begin{array}{l}
u_{1}^{\prime}(x)+1002 u_{1}(x)+1000 u_{2}^{2}(x)=0 \\
u_{2}^{\prime}(x)-u_{1}(x)+u_{2}(x)+u_{2}^{2}(x)=0 \\
u_{1}(0)=1, u_{2}(0)=1
\end{array}\right.
$$

The exact solution of Eq.(23) is given by $u_{1}(x)=e^{-2 x}, u_{2}(x)=e^{-x}$. The numerical results that are obtained using the proposed method are presented in Table 1, Table 2 and Table 3. Also, the graphical comparisons of the approximate and exact solutions are given in Figure 1 and Figure 2.

Table 1: Numerical comparison of the error functions $E_{1, N}$ and $E_{2, N}$ at the different values of $N$ for Example 2

\begin{tabular}{cccccccc}
\hline \multirow{2}{*}{$x$} & \multicolumn{2}{c}{ Chebyshev operational matrix method [13], $u_{1}$} & & \multicolumn{3}{c}{ The proposed method, $u_{1}$} \\
\cline { 2 - 4 } \cline { 6 - 7 } & $E_{1,6}$ & $E_{1,7}$ & $E_{1,8}$ & & $E_{1,6}$ & $E_{1,7}$ & $E_{1,8}$ \\
\hline \hline 0.2 & $0.453 \times 10^{-6}$ & $0.244 \times 10^{-7}$ & $0.312 \times 10^{-8}$ & & $0.147 \times 10^{-6}$ & $0.157 \times 10^{-7}$ & $0.787 \times 10^{-9}$ \\
0.4 & $0.614 \times 10^{-6}$ & $0.529 \times 10^{-7}$ & $0.326 \times 10^{-8}$ & & $0.376 \times 10^{-6}$ & $0.114 \times 10^{-7}$ & $0.338 \times 10^{-9}$ \\
0.6 & $0.107 \times 10^{-6}$ & $0.671 \times 10^{-7}$ & $0.223 \times 10^{-8}$ & & $0.742 \times 10^{-6}$ & $0.213 \times 10^{-7}$ & $0.931 \times 10^{-9}$ \\
0.8 & $0.139 \times 10^{-5}$ & $0.102 \times 10^{-6}$ & $0.196 \times 10^{-8}$ & & $0.289 \times 10^{-5}$ & $0.246 \times 10^{-6}$ & $0.117 \times 10^{-7}$ \\
1 & $0.530 \times 10^{-5}$ & $0.350 \times 10^{-6}$ & $0.203 \times 10^{-7}$ & & $0.163 \times 10^{-4}$ & $0.159 \times 10^{-4}$ & $0.137 \times 10^{-5}$ \\
\hline \multirow{2}{*}{$x$} & \multicolumn{2}{c}{ Chebyshev operational matrix method [13], $u_{2}$} & & \multicolumn{2}{c}{ The proposed method, $u_{2}$} \\
\cline { 7 - 8 } \cline { 6 - 8 } & $E_{2,6}$ & $E_{2,7}$ & $E_{2,8}$ & & $E_{2,6}$ & $E_{2,7}$ & $E_{2,8}$ \\
\hline \hline 0.2 & $0.167 \times 10^{-7}$ & $0.382 \times 10^{-10}$ & $0.143 \times 10^{-10}$ & & $0.311 \times 10^{-7}$ & $0.117 \times 10^{-8}$ & $0.440 \times 10^{-10}$ \\
0.4 & $0.217 \times 10^{-7}$ & $0.705 \times 10^{-9}$ & $0.214 \times 10^{-10}$ & & $0.190 \times 10^{-7}$ & $0.933 \times 10^{-9}$ & $0.377 \times 10^{-10}$ \\
0.6 & $0.222 \times 10^{-7}$ & $0.744 \times 10^{-9}$ & $0.221 \times 10^{-10}$ & & $0.145 \times 10^{-7}$ & $0.603 \times 10^{-9}$ & $0.311 \times 10^{-10}$ \\
0.8 & $0.101 \times 10^{-7}$ & $0.716 \times 10^{-9}$ & $0.180 \times 10^{-10}$ & & $0.334 \times 10^{-7}$ & $0.344 \times 10^{-9}$ & $0.154 \times 10^{-10}$ \\
1 & $0.518 \times 10^{-8}$ & $0.349 \times 10^{-9}$ & $0.202 \times 10^{-10}$ & & $0.577 \times 10^{-6}$ & $0.222 \times 10^{-7}$ & $0.389 \times 10^{-9}$ \\
\hline
\end{tabular}

Table 2: Numerical results of the maximum error $E_{1, N}$ at the different values of $N$ for Example 2

\begin{tabular}{ccccc}
\hline$N$ & 2 & 5 & 8 & 11 \\
$E_{1, N}$ & $0.303 \times 10^{0}$ & $0.144 \times 10^{-2}$ & $0.137 \times 10^{-5}$ & $0.465 \times 10^{-9}$ \\
\hline
\end{tabular}


Table 3: Numerical results of the maximum error $E_{2, N}$ at the different values of $N$ for Example 2

\begin{tabular}{ccccc}
\hline$N$ & 2 & 5 & 8 & 11 \\
$E_{2, N}$ & $0.319 \times 10^{-1}$ & $0.124 \times 10^{-4}$ & $0.389 \times 10^{-9}$ & $0.270 \times 10^{-12}$ \\
\hline
\end{tabular}
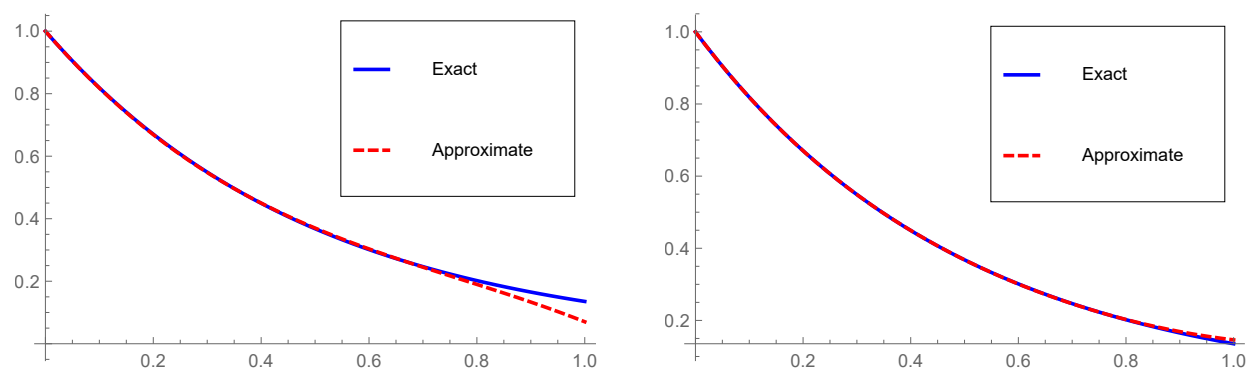

(a) $N=3$

(b) $N=4$

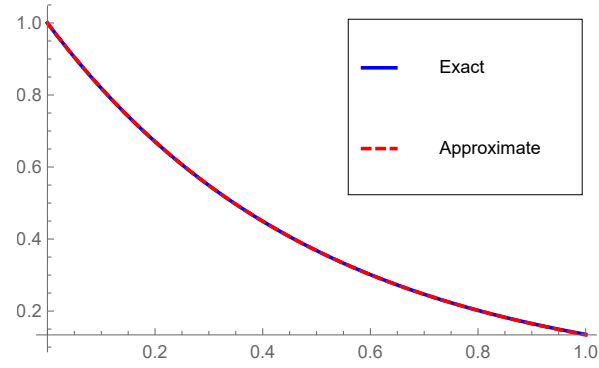

(c) $N=5$

Fig. 1: Graphical comparison of the exact and approximate solutions for $u_{1}$ when $N=3,4,5$ for Example 2

Example 3. Assume that the following differential equation system

$$
\begin{cases}u_{1}^{\prime \prime}(x)\left(1+u_{2}^{2}(x)\right)+u_{2}^{\prime}(x)\left(1+u_{1}(x)\right) & =g_{1}(x) \\ u_{2}^{\prime \prime}(x)\left(1+u_{1}^{2}(x)\right)+u_{1}^{\prime}(x)\left(1+u_{2}(x)\right) & =g_{2}(x) \\ u_{1}(0)=1, u_{2}(0)=1 & \end{cases}
$$

The exact solution of Eq.(24) is given by $u_{1}(x)=\sin x, u_{2}(x)=e^{x}$. Here, $g_{1}(x)=e^{x}-\sin x+e^{x} \sin x-e^{2 x} \sin x, g_{2}(x)=$ $e^{x}+\cos x+e^{x} \cos x+e^{x} \sin ^{2} x$. The numerical results that are obtained using the proposed method are presented in Table 4, Table 5 and Table 6. Also, the graphical comparisons of the approximate and exact solutions are given in Figure 3 and Figure 4.

\section{Competing interests}

The authors declare that they have no competing interests. 


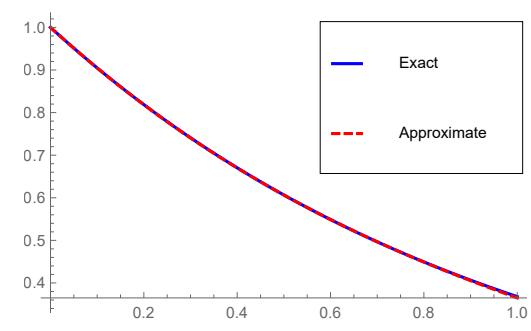

(a) $N=3$

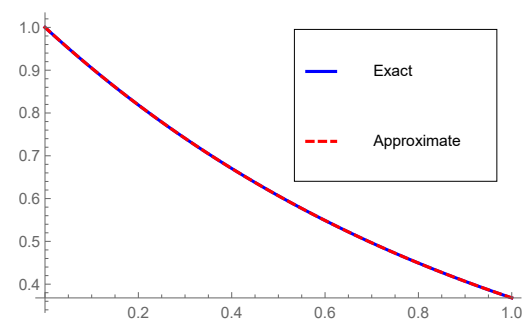

(b) $N=4$

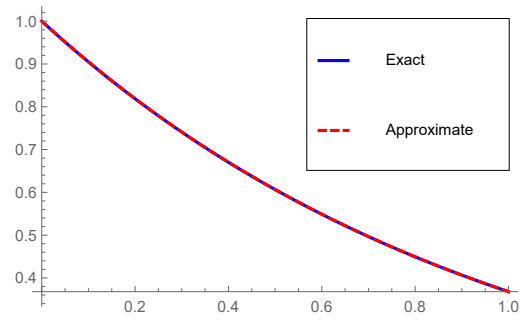

(c) $N=5$

Fig. 2: Graphical comparison of the exact and approximate solutions for $u_{2}$ when $N=3,4,5$ for Example 2

Table 4: Numerical comparison of the error functions $E_{1, N}$ and $E_{2, N}$ at the different values of $N$ for Example 3

\begin{tabular}{cccccccc}
\hline \multirow{2}{*}{$x$} & \multicolumn{3}{c}{$u_{1}$} & & \multicolumn{3}{c}{$u_{2}$} \\
\cline { 2 - 4 } \cline { 5 - 7 } & $E_{1,6}$ & $E_{1,7}$ & $E_{1,8}$ & & $E_{2,6}$ & $E_{2,7}$ & $E_{2,8}$ \\
\hline \hline 0.2 & $0.533 \times 10^{-7}$ & $0.527 \times 10^{-9}$ & $0.827 \times 10^{-10}$ & & $0.794 \times 10^{-7}$ & $0.309 \times 10^{-8}$ & $0.114 \times 10^{-9}$ \\
0.4 & $0.123 \times 10^{-6}$ & $0.736 \times 10^{-9}$ & $0.164 \times 10^{-9}$ & & $0.193 \times 10^{-6}$ & $0.627 \times 10^{-8}$ & $0.206 \times 10^{-9}$ \\
0.6 & $0.214 \times 10^{-6}$ & $0.589 \times 10^{-9}$ & $0.235 \times 10^{-9}$ & & $0.352 \times 10^{-6}$ & $0.918 \times 10^{-8}$ & $0.259 \times 10^{-9}$ \\
0.8 & $0.372 \times 10^{-6}$ & $0.706 \times 10^{-10}$ & $0.274 \times 10^{-9}$ & & $0.639 \times 10^{-6}$ & $0.117 \times 10^{-7}$ & $0.232 \times 10^{-9}$ \\
1 & $0.501 \times 10^{-5}$ & $0.116 \times 10^{-6}$ & $0.112 \times 10^{-7}$ & & $0.851 \times 10^{-5}$ & $0.400 \times 10^{-6}$ & $0.192 \times 10^{-7}$ \\
\hline
\end{tabular}

Table 5: Numerical results of the maximum error $E_{1, N}$ at the different values of $N$ for Example 3

\begin{tabular}{ccccc}
\hline$N$ & 2 & 5 & 8 & 11 \\
$E_{1, N}$ & $0.158 \times 10^{0}$ & $0.294 \times 10^{-4}$ & $0.112 \times 10^{-7}$ & $0.255 \times 10^{-12}$ \\
\hline
\end{tabular}

Table 6: Numerical results of the maximum error $E_{2, N}$ at the different values of $N$ for Example 3

\begin{tabular}{ccccc}
\hline$N$ & 2 & 5 & 8 & 11 \\
$E_{2, N}$ & $0.218 \times 10^{0}$ & $0.123 \times 10^{-3}$ & $0.192 \times 10^{-7}$ & $0.877 \times 10^{-12}$ \\
\hline
\end{tabular}

\section{Authors' contributions}

All authors have contributed to all parts of the article. All authors read and approved the final manuscript. 


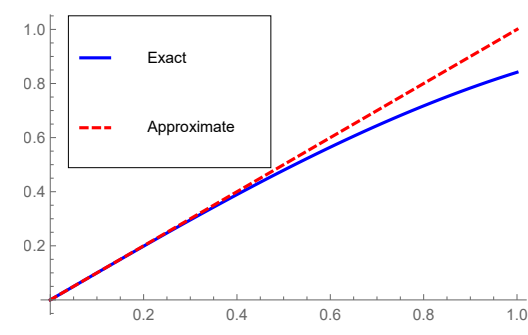

(a) $N=2$

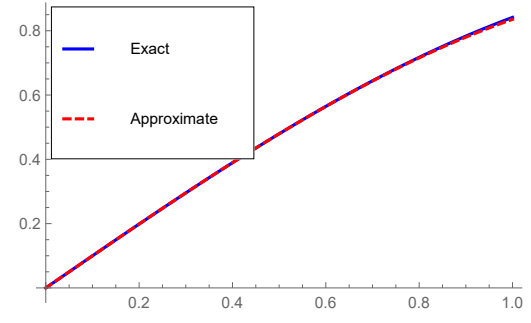

(b) $N=3$

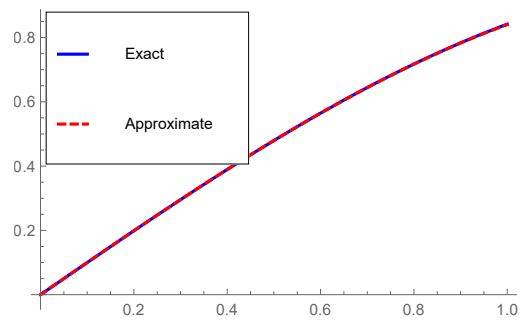

(c) $N=4$

Fig. 3: Graphical comparison of the exact and approximate solutions for $u_{1}$ when $N=2,3,4$ for Example 3

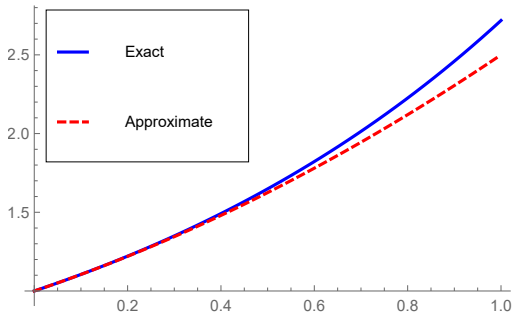

(a) $N=2$

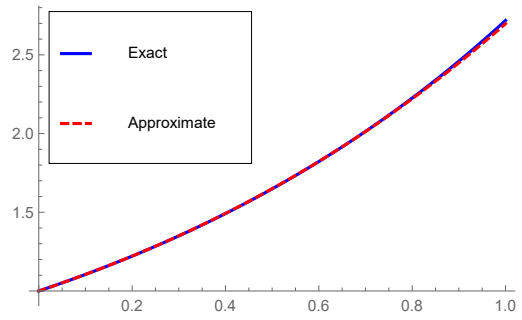

(b) $N=3$

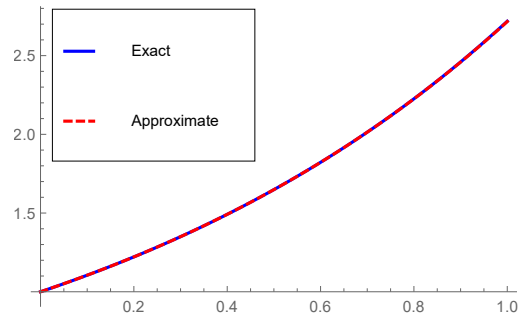

(c) $N=4$

Fig. 4: Graphical comparison of the exact and approximate solutions for $u_{2}$ when $N=2,3,4$ for Example 3 


\section{Conclusions}

In this paper, the Fibonacci collocation method is used for solving a class of systems of nonlinear differential equations. For the accuracy and efficiency of the method, three different examples are presented. The obtained results are compared with ones obtained with Chebyshev operational matrix method. As a result of these comparisons, it can be said that the method is very effective for approximately solving systems of nonlinear differential equations.

\section{References}

[1] Kumbinarasaiah, S., Raghunatha, K. R., The applications of hermite wavelet method to nonlinear differential equations arising in heat transfer, International Journal of Thermofluids, 9, 2021: 100066.

[2] Odibat, Z., An improved optimal homotopy analysis algorithm for nonlinear differential equations, Journal of Mathematical Analysis and Applications, 2020, 488(2), 124089.

[3] Gurbuz, B., A computational approach for solving second-order nonlinear ordinary differential equations by means of Laguerre series, Bitlis Eren Üniversitesi Fen Bilimleri Dergisi, 9(1), 2020: 78-84.

[4] He, J. H., Latifizadeh, H., A general numerical algorithm for nonlinear differential equations by the variational iteration method, International Journal of Numerical Methods for Heat \& Fluid Flow, 2020, 30(11),4797-4810.

[5] Odibat, Z., An optimized decomposition method for nonlinear ordinary and partial differential equations, Physica A: Statistical Mechanics and its Applications, 2020, 541, 123323.

[6] Mirzaee, F., Hoseini, S. F., Solving systems of linear Fredholm integro-differential equations with Fibonacci polynomials, Ain Shams Engineering Journal, (2014), 5(1), 271-283.

[7] Kurt, A., Yalçinbas, S., Sezer, M., Fibonacci collocation method for solving high-order linear Fredholm integro-differentialdifference equations, International Journal of Mathematics and Mathematical Sciences, (2013), 2013.

[8] Mirzaee, F., Hoseini, S. F., A new collocation approach for solving systems of high-order linear Volterra integro-differential equations with variable coefficients, Applied Mathematics and Computation, (2017), 311, 272-282.

[9] Kurt, A., Yalçinbas, S., Sezer, M., Fibonacci collocation method for solving linear differential-difference equations, Mathematical and Computational Applications, (2013), 18(3), 448-458.

[10] Mirzaee, F., Hoseini, S. F., Solving singularly perturbed differential-difference equations arising in science and engineering with Fibonacci polynomials, Results in Physics, (2013), 3, 134-141.

[11] Falcon, S., Plaza, Á., The k-Fibonacci sequence and the Pascal 2-triangle, Chaos, Solitons \& Fractals (2007), 33.1, 38-49.

[12] Falcon, S., Plaza, Á., On k-Fibonacci sequences and polynomials and their derivatives, Chaos, Solitons \& Fractals, (2009), 39.3, 1005-1019.

[13] Öztürk, Y., Numerical solution of systems of differential equations using operational matrix method with Chebyshev polynomials, Journal of Taibah University for Science, (2018), 12.2, 155-162. 